\title{
The Lamellar-Disorder Interface: One-Dimensional Modulated Profiles
}

\author{
Simon Villain-Guillot * and David Andelman \\ School of Physics and Astronomy \\ Raymond and Beverly Sackler Faculty of Exact Sciences \\ Tel-Aviv University \\ Ramat-Aviv 69978, Tel-Aviv, Israel
}

\begin{abstract}
We study interfacial behavior of a lamellar (stripe) phase coexisting with a disordered phase. Systematic analytical expansions are obtained for the interfacial profile in the vicinity of a tricritical point. They are characterized by a wide interfacial region involving a large number of lamellae. Our analytical results apply to systems with one dimensional symmetry in true thermodynamical equilibrium and are of relevance to metastable interfaces between lamellar and disordered phases in two and three dimensions. In addition, good agreement is found with numerical minimization schemes of the full free energy functional having the same one dimensional symmetry. The interfacial energy for the lamellar to disordered transition is obtained in accord with mean field scaling laws of tricritical points.
\end{abstract}

${ }^{*}$ Permanent address:

Groupe de Physique Statistique

Université de Cergy-Pontoise

BP 8428, 95806 Cergy-Pontoise Cedex, France 


\section{INTRODUCTION}

Some equilibrium phases of matter exhibit spatial modulations in one or more of their local properties (local order parameter) and hence are called modulated phases [1]. Modulated phases can be found in a variety of physical and chemical systems such as diblock copolymers, magnetic garnet films, ferrofluids, adsorbates on surfaces of metals, superconductors (Type I) and semiconductors. Although these systems all have spatial modulations in their order parameter, the microscopic origin of these modulations is very different.

Usually modulated phases manifest either a one dimensional packing of sheets (the socalled lamellar or stripe phase), two-dimensional packing of cylinders with an hexagonal symmetry or a cubic packing of spheres or other, more complex, bi-continuous superstructures with cubic symmetry. The origin of the preferred length scale of the modulations can be explained in many cases by a competition between two type of interactions: a short-range interaction which tends to make the system more homogeneous together with a long-range one, or a non-local one, which prefers proliferation of domain walls. For example, in the magnetic films, the dipole-dipole interaction has the latter effect.

These two opposing interactions are responsible in the above systems for the appearance of spatially modulated phases having an optimal non-zero wave-vector. Depending on the range of the thermodynamical variables, instead of having the usual segregation of the system into two homogeneous phases (such as coexisting liquid and gas phases, or two coexisting polymer phases), the system self-organizes into alternating domains. Those domains are associated with spatially modulated order parameter (polarization, magnetization, relative concentration), and can be regarded as unit cells of super-structures of lamellar, hexagonal or cubic symmetry. Depending on the system in mind, the domain length scale can be as small as one hundred angstroms or as large as centimeters [1]. In contrast to periodic crystalline structures, the periodicity in modulated phases can be tuned by varying external fields such as pressure, temperature and electric or magnetic fields.

In this paper we would like to present analytical results related to interfaces in modulated phases. When the modulations are relatively weak an analytical expansion of the interfacial profile can be obtained systematically. In particular, we calculate the profile of the lamellar-to-disordered interface for a model free energy where spatial modulations are restricted along one dimension. Physical systems can create more complicated modulations in two- and three dimensions. In many cases, the lamellar-to-disordered interface addressed here can be shown to be only metastable [2, 3] and not in true thermodynamical equilibrium. Nevertheless, their systematic study can be useful to understand nucleation and growth in e.g., polymeric systems with slow dynamics. In addition, the rather simplified lamellar-disordered interface can be generalized to other situations.

Our analytical results are valid in the vicinity of a tricritical point which occurs for asymmetric profiles in our strictly one-dimensional model. Close to the tricritical point it is possible to decouple the two characteristic length scales which appears in the model : the period associated with the modulated phase and the width of the interface. Thus, it 
is possible to compute analytically the leading terms of the interfacial energies.

The obtained results presented here are in good agreement with recent work by Netz et al [2] where numerical minimization of a similar free energy functional yielded detailed structure of interfaces between various two-dimensional modulated phases.

\section{THE MODEL AND ITS PHASE DIAGRAM}

A phenomenological free energy $F$ similar to the one introduced in Ref. [2] is used hereafter in order to allow us to study one-dimensional profiles between lamellar and disordered phases.

$$
F=2\left(\nabla^{2} \phi\right)^{2}-2(\nabla \phi)^{2}+\frac{t}{2} \phi^{2}+\frac{1}{4} \phi^{4}
$$

This mean-field free energy is a modified Ginzburg-Landau (GL) expansion in the order parameter $\phi(x)$, which varies between $\phi=1$ for A-rich domains to $\phi=0$ for B-rich domains. The last two terms in $F$ are the usual terms appearing in the Landau expansion with $t=\left(T-T_{c}\right) / T_{c}$ being the reduced temperature and the coefficient of the fourth order term is chosen to be $1 / 4$ without loss of generality. For an order parameter without spatial modulations they define our disorder free energy, $F_{D}$ (within the Landau expansion):

$$
F_{D}=\frac{t}{2} \phi^{2}+\frac{1}{4} \phi^{4}
$$

The main deviation from the regular GL expansion can be seen in the first two terms of (1). The coefficient of the gradient square term is negative (favoring modulations), whereas the coefficient of the Laplacian square term is positive (preventing strong modulations) and playing the role of a homogenizing interaction. An illustrative example can be a diblock copolymer melt where each polymer is composed of two blocks, A and B, which would like to phase separate. However, since the A and B chains are chemically linked forming one polymer chain, the system can only undergo a micro-phase separation resulting in a spatial modulation in the local $\mathrm{A} / \mathrm{B}$ relative concentration.

In order to construct the phase diagram, we compare the mean-field free energy $F_{D}$ associated with the disordered phase $\left(\phi=\right.$ const.) with the free energy $F_{L}$ of a phase described by a modulating $\phi(\vec{r})$. Our approach involves several simplifying assumptions: In the vicinity of a critical point (weak segregation limit), an analytical expansion of the free energy in powers of the small order parameter (Landau expansion) can be justified. Since a mean-field approximation is employed, critical fluctuations corrections are omitted [4.5]. Furthermore, only the most dominant modulation mode (the so-called single-mode approximation) is considered because the amplitude of the fluctuations of other $q$-modes grows more slowly in this region as was shown by Brazovskii [4]. Finally, only onedimensional modulations of $\phi(\vec{r})=\phi(x)$ along an arbitrary chosen $x$ direction will be explored. This reduces the problem into an effective one-dimensional problem. It applies 
to interfaces where the lamellae are themselves parallel to the lamellar - disorder interface. We further discuss the validity and limitations of our assumptions in the last section.

$$
\phi=\phi_{o}+2 \phi_{q} \cos (q x)
$$

where $\phi_{o}$ is the mean spatial order parameter (the average A/B relative concentration) and $\phi_{q}$ is the amplitude of the $q$-mode modulation.

The most dominant mode is obtained by minimizing the free energy with respect to the $q$-vector. Within the single mode approximation this leads to $q^{*}=1 / \sqrt{2}$ with our choice of simple coefficients in (1). This is the wave-vector characterizing the periodicity of the super-structure, $2 \pi / q^{*}$. Inserting $\phi(x)$ of the dominant mode from (3) into (1) we obtain the lamellar free energy

$$
F_{L}=\frac{t}{2} \phi_{o}^{2}+\frac{1}{4} \phi_{o}^{4}+\left(t-1+3 \phi_{o}^{2}\right) \phi_{q}^{2}+\frac{3}{2} \phi_{q}^{4}
$$

The disorder free energy $F_{D}(2)$ is simply given by setting $\phi_{q}^{2}=0$ in the above expression for $F_{L}$.

Since this expression is symmetric with respect to $\phi_{o} \rightarrow-\phi_{o}$ only the $\phi_{o} \geq 0$ region can be considered without loss of generality. We then apply the variational principle with respect to $\phi_{q}$. This enables us to integrate over $\phi_{q}$ - one of the two order parameters. Since the amplitude of the modulation minimizing $F_{L}$ is :

$$
\phi_{q}^{2}=\frac{1-t}{3}-\phi_{o}^{2}
$$

the necessary condition for the existence of this modulated phase is $\phi_{q}^{2} \geq 0$, hence

$$
\frac{1-t}{3}-\phi_{o}^{2} \geq 0
$$

Substituting $\phi_{q}^{2}$ from (5) into (4) gives the free energy for the lamellar phase

$$
F_{L}=-\frac{(t-1)^{2}}{6}+\left(1-\frac{t}{2}\right) \phi_{o}^{2}-\frac{5}{4} \phi_{o}^{4}
$$

In the region bounded by inequality (6) and close to the critical point, the free energy is a convex function of $\phi_{o}$. Therefore, the second-order phase transition line separating the order and the disordered phases is given by:

$$
\phi_{o}^{2}=\frac{1-t}{3}
$$

showing an upward shift of the critical temperature $t_{c}=1$ from its usual value for the GL model, $t_{c}=0$. A tricritical point appears at a temperature $t_{t c p}=\frac{3}{4}$, for which $\delta^{2} F_{L} / \delta \phi_{o}^{2}=$ 
0. Below this temperature, the free energy is concave; the system therefore prefers to phase separate. Equation (5) gives the value of the order parameter (concentration) at the tricritical point, $\phi_{T}^{2}=\phi^{2}\left(t_{t c p}\right)=\frac{1}{12}$.

In Figs. 1 and 2 the phase diagram is plotted as function of the reduced temperature - average concentration $\left(t, \phi_{o}\right)$, and as function of the chemical potential - reduced temperature $(\mu, t)$, respectively. The phase diagram is calculated numerically within the single mode approximation by comparing the lamellar free energy $F_{L}$ with the disorder one, $F_{D}$. We note that the phase diagram in the vicinity of the tricritical point resembles that of the BEG (Blume-Emery-Griffiths) spin one model [7]. The BEG model also has two order parameters and exhibits a similar behavior: a first order phase transition line meets a second-order one at a tricritical point.

In the vicinity of the tricritical point (but inside the two phase region) we are still in the weak segregation limit. Hence, a systematic expansion can be performed as function of a new reduced temperature $\varepsilon$, measuring the distance from the tricritical point: $\varepsilon=$ $t_{t c p}-t>0$. We then get :

$$
F_{L}=\frac{t}{2} \phi_{0}^{2}+\frac{1}{4} \phi_{0}^{4}-\frac{3}{2}\left(\phi_{T}^{2}+\frac{\varepsilon}{3}-\phi_{0}^{2}\right)^{2}
$$

This equation can be expanded in terms of $\eta_{D}$ and $\eta_{L}$, such that $\phi(\varepsilon)=\phi_{T}+\eta_{D}(\varepsilon)$ for the disordered (isotropic) phase and $\phi(\varepsilon)=\phi_{T}+\eta_{L}(\varepsilon)$ for the lamellar phase. In the two-phase coexistence region, the boundaries are given by the common tangent method :

$$
\mu_{D}=\mu_{L}=\mu
$$

together with

$$
G_{L} \equiv F_{L}-\mu \phi_{L}=G_{D} \equiv F_{D}-\mu \phi_{D}
$$

where $F_{L}$ and $F_{D}$ are expressed in term of $\eta_{D}$ and $\eta_{L}$, respectively.

$$
\begin{aligned}
& F_{L}=\frac{t}{2} \phi_{T}^{2}+\frac{1}{4} \phi_{T}^{4}-\frac{\varepsilon^{2}}{6}+\left(\frac{5}{6}+\varepsilon\right) \phi_{T} \eta_{L}+\frac{\varepsilon}{2} \eta_{L}^{2}-5 \phi_{T} \eta_{L}^{3}-\frac{5}{4} \eta_{L}^{4} \\
& F_{D}=\frac{t}{2} \phi_{T}^{2}+\frac{1}{4} \phi_{T}^{4}+\left(\frac{5}{6}-\varepsilon\right) \phi_{T} \eta_{D}+(1-\varepsilon) \frac{\eta_{D}^{2}}{2}+\phi_{T} \eta_{D}^{3}+\frac{1}{4} \eta_{D}^{4}
\end{aligned}
$$

Relation (10) enables us to write $\eta_{D}$ as an expansion in $\eta_{L}$ up to third order in $\varepsilon$ :

$$
\eta_{D}=2 \phi_{T} \varepsilon+\phi_{T} \varepsilon^{2}+\varepsilon \eta_{L}-15 \phi_{T} \eta_{L}^{2}-\frac{2}{3} \phi_{T} \varepsilon^{3}-5 \eta_{L}^{3}
$$

and relation (11) leads to $\eta_{L}$ up to third order in $\varepsilon$ :

$$
\eta_{L}=\phi_{T}\left(-\frac{2}{5} \varepsilon+\frac{1}{25} \varepsilon^{2}+\frac{13}{250} \varepsilon^{3}\right)
$$


Therefore :

$$
\eta_{D}=\phi_{T}\left(2 \varepsilon+\frac{2}{5} \varepsilon^{2}-\frac{14}{25} \varepsilon^{3}\right)
$$

In terms of newly defined order parameters: $\phi_{ \pm} \equiv\left(\phi_{D} \pm \phi_{L}\right) / 2$, we get

$$
\begin{aligned}
& \phi_{+}=\frac{1}{2}\left(\phi_{D}+\phi_{L}\right)=\phi_{T}+\frac{1}{2}\left(\eta_{D}+\eta_{L}\right)=\phi_{T}\left(1+\frac{4}{5} \varepsilon+\frac{11}{50} \varepsilon^{2}-\frac{127}{250} \varepsilon^{3}\right) \\
& \phi_{-}=\frac{1}{2}\left(\phi_{D}-\phi_{L}\right)=\frac{1}{2}\left(\eta_{D}-\eta_{L}\right)=\phi_{T}\left(\frac{6}{5} \varepsilon+\frac{9}{50} \varepsilon^{2}-\frac{153}{250} \varepsilon^{3}\right) \\
& \phi_{q}^{2}=\frac{1-t}{3}-\phi_{L}^{2}=\frac{2}{5} \varepsilon-\frac{\varepsilon^{2}}{50}-\frac{3}{500} \varepsilon^{3}
\end{aligned}
$$

Note that at the tricritical point, the slopes of the two phase transition lines given by Eq.(8) and Eq.(16) are equal as has been noticed by the original mean-field work of Ref. [7].

\section{THE LAMELLAR-DISORDER INTERFACIAL PROFILE}

In a simple condensation transition, the interface width between the two homogeneous phases, $\xi \sim t^{-1 / 2}$, has the same scaling behavior as the typical concentration fluctuations of the homogeneous phase. In a modulated phase, a second characteristic length scale exists and has to be considered together with this correlation length. This is the wavelength of the super structure, $q^{*}$. In order to compute analytically the profile of the order parameter for the disorder-lamellar interface close to the tricritical point, where the two length scales are expected to be uncorrelated, we propose the following ansatz:

$$
\phi(x)=\phi_{+}+\delta \phi(x) \quad \text { where } \quad \delta \phi(x)=\phi_{-} f(x)+\phi_{q} g(x) \cos \left(q^{*} x\right)
$$

Far from the interface, in the disordered region, $f(x)=1$ and $g(x)=0$, whereas in the lamellar phase region, $f(x)=-1$ and $g(x)=2$. These are the boundary conditions for the differential equations of the interfacial profile. The slowly spatially varying functions

$f(x)$ and $g(x)$ play the role of the two order parameters. The difference in the scaling of $\phi_{-}$and $\phi_{q}$ with $\varepsilon$ close to the tricritical point, enables us to decouple the behavior of these two functions.

Expanding the chemical potential up to the third order in $\phi_{-}$, we find :

$$
\mu-t \phi_{+}-\phi_{+}^{3}=\alpha \phi_{-}+3 \phi_{+} \phi_{-}^{2}+\phi_{-}^{3}
$$

where $\alpha$ is define as:

$$
\alpha \equiv \frac{\partial^{2} F_{D}}{\partial \phi_{D}^{2}}=t+3 \phi_{+}^{2}=1-\frac{3}{5} \varepsilon+\frac{27}{100} \varepsilon^{2}-\frac{\varepsilon^{3}}{6}
$$


Incorporating ansatz (18) into $G_{L}=F_{L}-\mu \phi_{L}$, we get :

$$
\begin{array}{r}
F_{L}-\mu \phi_{L}=G\left(\phi_{+}\right)+\frac{\alpha}{2}(\delta \phi)^{2}+\phi_{+}(\delta \phi)^{3}+\frac{1}{4}(\delta \phi)^{4}-\left(\alpha \phi_{-}+3 \phi_{+} \phi_{-}^{2}+\phi_{-}^{3}\right) \delta \phi+ \\
2\left\{f^{\prime \prime} \phi_{-}+\phi_{q}\left[\left(g^{\prime \prime}-\left(q^{*}\right)^{2} g\right) \cos \left(q^{*} x\right)-2 q^{*} g^{\prime} \sin \left(q^{*} x\right)\right]^{2}-2\left(f^{\prime} \phi_{-}+\phi_{q}\left(g^{\prime} \cos \left(q^{*} x\right)-q g \sin \left(q^{*} x\right)\right)\right\}^{2}\right.
\end{array}
$$

Close to the tricritical point, the two different length scales behave differently. The profile is characterized by the width of the interface, $\xi$, which should diverge at $t=t_{t c p}$. However, in the same limit, the modulation wavelength, $2 \pi / q^{*}$, remains unchanged, as can be checked from the numerical calculations (see Fig. 5). Therefore, taking the average of $F_{L}-\mu \phi_{L}$ over one period, yields :

$$
\begin{gathered}
\left\langle F_{L}-\mu \phi_{L}\right\rangle= \\
G\left(\phi_{+}\right)+2 \phi_{-}^{2}\left[\left(f^{\prime \prime}\right)^{2}-\left(f^{\prime}\right)^{2}\right]+\phi_{q}^{2}\left[\left(g^{\prime \prime}-\frac{1}{2} g\right)^{2}+g^{\prime 2}-\frac{1}{2} g^{2}\right]+\frac{\alpha}{2} \phi_{-}^{2} f^{2}+\frac{\alpha}{4} \phi_{q}^{2} g^{2}+ \\
\phi_{+} \phi_{-}^{3} f^{3}+\frac{3}{2} \phi_{q}^{2} \phi_{-} \phi_{+} f g^{2}+\frac{1}{4} \phi_{-}^{4} f^{4}+\frac{3}{4} \phi_{q}^{2} g^{2} f^{2} \phi_{-}^{2}+\frac{3}{32} \phi_{q}^{4} g^{4}-\left(\alpha \phi_{-}+3 \phi_{+} \phi_{-}^{2}\right) \phi_{-} f
\end{gathered}
$$

The variational principle with respect to $f(x)$ leads to the relation :

$$
\alpha f+3 \phi_{+} \phi_{-} f^{2}+\frac{3}{2} \frac{\phi_{q}^{2}}{\phi_{-}} \phi_{+} g^{2}+\frac{3}{2} \phi_{q}^{2} f g^{2}+\phi_{-}^{2} f^{3}=\alpha+3 \phi_{+} \phi_{-}
$$

Using expressions (17) for $\phi_{+}, \phi_{-}$and $\phi_{q}$, we get up to the first order in $\varepsilon$ :

$$
f(x)=1-\frac{1}{2} g^{2}+\frac{9}{10} g^{2}\left(\frac{g^{2}}{4}-1\right) \varepsilon
$$

This relation enables us to find, when applying the variational principle with respect to $g(x)$, the following differential equation :

$$
4 g^{\prime \prime}=\frac{\alpha-1}{2} g+3 f g \phi_{-} \phi_{+}+\frac{3}{2} \phi_{-}^{2} f^{2} g+\frac{3}{8} \phi_{q}^{2} g^{3}
$$

which can be rewritten at the lowest order in $\varepsilon$ as :

$$
\frac{20}{3} g^{\prime \prime}(x)=\left(g-g^{3}+\frac{3}{16} g^{5}\right) \varepsilon^{2}
$$

Taking into account the boundary conditions for $g(x)$, the analytical solution of this differential equation is found to be :

$$
g(U)=\frac{2 \mathrm{e}^{-U}}{\sqrt{1+\mathrm{e}^{-2 U}}}=\frac{2}{\sqrt{1+\mathrm{e}^{2 U}}}
$$


where $U(x)=\frac{1}{2} \sqrt{\frac{3}{5}}\left(x-x_{0}\right) \varepsilon$, and $x_{0}$ can be set to zero without lost of generality. Using Eq. (24) we can now obtain for $f$

$$
f(U)=\tanh (U)-\frac{9}{10} \cosh ^{-2}(U) \varepsilon
$$

The two functions $f(x)$ and $g(x)$ determine the interfacial profile $\phi(x)$ close to the tricritical point and are plotted in Fig. 3 and Fig. 4. The width of the interface scales as $\xi \sim \varepsilon^{-1}$. Namely, the correlation length exponent $\nu=1$ is recovered, in agreement with the scaling laws associated with the tricritical point [9]. We note that the main reason it is possible to decouple the differential equations for $f$ and $g$ (to lowest order in $\varepsilon$ ) is due to the different scaling of $\phi_{q}$ and $\phi_{-}$with respect to $\varepsilon$ close to the tricritical point [see Eq. (17)].

\section{THE DISORDER-LAMELLAR INTERFACIAL TENSION}

The interfacial tension $\gamma$, is defined as the excess of free energy due to the interface. It can be written as :

$$
\gamma=\int \Gamma(x) d x=\int\left[G-\frac{G_{D}+G_{L}}{2}\right] d x=\int\left[F-\frac{F_{D}+F_{L}}{2}-\mu \delta \phi(x)\right] d x
$$

Since up to second order in $\varepsilon$, the even part of $\Gamma(x)$ vanishes, we have to keep terms in $\Gamma$ up to third order in $\varepsilon$.

$$
\begin{aligned}
\Gamma=2 \phi_{q}^{2}\left(g^{\prime}\right)^{2} & +\frac{\alpha-1}{4} \phi_{q}^{2}\left(g^{2}-2\right)+3 \phi_{q}^{2} \phi_{-} \phi_{+}\left(\frac{f g^{2}}{2}+1\right)+\frac{3}{2} \phi_{q}^{2} \phi_{-}^{2}\left(\frac{f^{2} g^{2}}{2}-1\right) \\
& +\frac{3}{4} \phi_{q}^{4}\left(\frac{g^{4}}{8}-1\right)-\phi_{-}^{2}\left(\alpha+3 \phi_{+} \phi_{-}\right) f+\frac{\alpha}{2} \phi_{-}^{2}\left(f^{2}-1\right)+\phi_{-}^{3} \phi_{+} f^{3}
\end{aligned}
$$

This expression can be written as function of $f(x)$ only :

$$
\Gamma=\left(1-f^{2}\right)\left(\frac{3}{50}-\frac{\alpha}{2} \phi_{-}^{2}+3 \phi_{q}^{2} \phi_{-} \phi_{+}-\frac{3}{2} \phi_{q}^{2} \phi_{-}^{2}-\frac{3}{8} \phi_{q}^{4}\right)+\text { odd terms }
$$

and to third order in $\varepsilon$

$$
\Gamma=\left(\frac{27}{250} \cosh ^{-2}(U)+\text { odd terms }\right) \varepsilon^{3}+O\left(\varepsilon^{4}\right)
$$

where the odd terms in the above equations are odd with respect to $x \rightarrow-x$ and will vanish upon integration over $x$, yielding 


$$
\gamma=\frac{18}{25} \sqrt{\frac{3}{5}} \varepsilon^{2}
$$

This result corresponds to the expected tricritical exponent $\mu=2$, in agreement with the hyperscaling relation $\mu=(d-1) \nu$, as the upper critical dimension for the tricritical point is $d=3$ [9].

We note that in order to have the full expression of $f(x)$ up to first order in $\varepsilon$, one needs to expand $g(x)$ up to first order in $\varepsilon$. Indeed, if $g(x)=g_{o}(x)+g_{1}(x) \varepsilon$, then $f(x)$ can be written as :

$$
f(x)=1-\frac{1}{2} g^{2}+\frac{9}{10} g^{2}\left(\frac{g^{2}}{4}-1\right) \varepsilon-g_{o}(x) g_{1}(x)=f_{o}(x)+f_{1}(x) \varepsilon .
$$

Nevertheless, only the leading order terms of $g(x)$ and $f(x)$ are required to compute the interfacial tension.

Numerical studies of this one-dimensional interface without assuming single-mode dominance give results in good agreement with the analytical calculation presented above. We have discretized the order parameter $\phi$ and chosen the grid spacing to be much smaller than $1 / q^{*}$ (typically 180 grid points per period). The free energy (何 can then be minimized using the conjugate gradient method.

The free energies for the disordered (isotropic) and lamellar phases (without using the single mode approximation) are computed in order to determine the chemical potential for the coexisting phases. Then, we compute the free energy associated with the interface, in an interval much bigger than the expected interface length, in order to neglected boundary effect. The boundary conditions are taken as reflecting (Dirichlet) boundary conditions. The same procedure of Ref. [2] using Eq. (29) is employed to calculate the interfacial energies. For small $\varepsilon=t_{t c p}-t$, the optimum wave vector is still $q^{*}=1 / \sqrt{2}$, within a good precision. The profiles of the interface for $\varepsilon=t_{t c p}-t=0.35$ and $\varepsilon=0.03$ are shown in Figs. 5 and 6, respectively.

The interfacial tension is plotted on Fig. 6 as a function of $t_{t c p}-t$. The log-log plot in $6 \mathrm{a}$ as well as the $\gamma^{1 / 2}$ plot in $6 \mathrm{~b}$ manifests clearly that our analytical result are: (i) in good agreement with the numerical results obtained from the full minimization of the interfacial free energy without assuming the single mode dominance; and (ii) that indeed we obtained the expected scaling behavior of the tricritical point.

\section{DISCUSSION AND CONCLUSIONS}

In this paper we have derived analytical expressions for the interface profiles between a simple modulated phase with a lamellar symmetry and a disordered one. Our results are valid close to the tricritical point since we assume that the modulated phase is characterized by its dominant $q$-mode in the bulk. This assumption is found to be compatible with the scaling laws close to the tricritical point. Our analytical (but approximated) 
results are in accord with numerical minimization of the full free energy functional having the same one-dimensional symmetry.

Unlike simple interfaces between two coexisting homogeneous phases, here the interface depends on two separate length scales. Close to the tricritical point, the interface width $\xi$ diverges and hence gets decoupled from the periodicity of the lamellar phase. It is interesting to note that in this case, the interfacial region can involve quite a number of lamellae periods as is shown in Figs. 4 and 5. For $\varepsilon=0.03$ the interfacial region spans over 100 lamellae.

Our results should be compared with the ones obtained by Fredrickson and Binder [3] since they obtained a similar envelope equation for the profile (eq. (27) for $g$ in our notation). In Ref. [3] the metastable interface close to a symmetric critical point is considered. Due to the symmetry, they have $f=0$ (in our notation). The high order terms $\left(\phi^{5}\right)$ in their profile equation arises from the first-loop correction (order $\phi^{6}$ ) in their mean-field effective Hamiltonian. In our case, due to the asymmetry, the coupling of $g$ to $f \neq 0$ generates the high order terms even within mean field theory. Needless to say that the scaling of the correlation length and surface tension is different in the two models because of the difference between critical and tricritical behavior.

It will be interesting to try to obtain experimental data on lamellar to disorder interfaces close to the tricritical point. Unfortunately, in many experimental realizations the one-dimensional phase diagram studied here is modified. The lamellar to disorder transition becomes metastable since it is pre-empted close to the tricritical point by phases with hexagonal and cubic symmetry [2]. Then, the lamellar-to-disordered interfaces can exist in thermodynamical equilibrium but only far from the tricritical point. Close to the tricritical point the lamellar to disordered transition describes only a metastable situation which is of importance, for example, for systems with slow dynamics like copolymer mixtures [3, 10]. We hope the systematic expansion we present can be applied to other more complicated situations where it is also possible to decouple the important length scales in the system.

\section{Acknowledgment}

We would like to thank J. Beckhöfer, E. Meron, R. Netz, M. Schick and B. Widom for discussions and comments. In particular, we thank T. Garel for very useful remarks and for bringing Ref. [3] to our attention. This work was partially supported by a grant from the United States-Israel Binational Science Foundation under grant No. 94-00291. One of us (SVG) thanks the French Ministry of Foreign Affairs for a research fellowship. 


\section{REFERENCES}

[1] M. Seul and D. Andelman, Science 267, 476 (1995).

[2] R. R. Netz, D. Andelman and M. Schick, Phys. Rev. Lett. 79, 1058 (1997).

[3] G. H. Fredrickson and K. Binder, J. Chem. Phys. 91, 7265 (1989).

[4] A. Brazovskii, Zh. Eksp. Teor. Fiz. 68, 175 (1975); [Sov. Phys. JETP 41, 85, (1975)].

[5] T. Garel and S. Doniach, Phys. Rev. B 26, 325 (1982).

[6] G. Grinstein and R. A. Pelcovits, Phys. Rev. A 26, 915 (1982).

[7] M. Blume, V.J. Emery and R.B. Griffiths, Phys. Rev. A 4, 1071, (1971).

[8] D. Andelman, F. Brochard and J.-F. Joanny, J. Chem. Phys. 86, 3673 (1987).

[9] I.D. Lawrie and S. Sarbach, "Theory of Tricritical Points" in Phase Transitions and Critical Phenomena, Vol. 9, C. Domb and M.S. Green editors, (McGraw-Hill, New York).

[10] P. C. Hohenberg and J. B. Swift, Phys. Rev. E 52, 1828 (1995). 


\section{FIGURE CAPTIONS}

Fig. 1 The calculated phase diagram from the modulated phase free energy $F_{L}$ as function of the reduced temperature - average order parameter $\left(t, \phi_{o}\right)$. The phase diagram is symmetric with respect to $\phi_{o} \rightarrow-\phi_{o}$. The two symmetric tricritical points are denoted by tcp. Below the tcp temperature, there are two regions of coexistence of the lamellar phase and disordered phase $\left(\mathrm{L}+\mathrm{D}_{1}\right)$ and $\left(\mathrm{L}+\mathrm{D}_{2}\right)$. At even lower temperatures, the lamellar phase disappears. We then recover the usual coexistence region between two different disordered phases: $\mathrm{D}_{1}+\mathrm{D}_{2}$ [8].

Fig. 2 Same as in Fig. 1 but as function of the chemical potential - reduced temperature $(\mu, t)$. The continuous line is a second-order transition line. The dashed line is a firstorder one. They meet at two tricritical points $(t c p)$. The two transition lines separate the lamellar phase region from the disordered region. The phase diagram is symmetric with respect to $\mu \rightarrow-\mu$. The $\mu=0$ first order transition line meets the two other first order lines at a triple point where all three phases: $\mathrm{L}, \mathrm{D}_{1}$ and $\mathrm{D}_{2}$ coexist.

Fig. 3 (a) Analytical profiles of the two functions $f(x)$ and $g(x)$ for $\varepsilon=t_{t c p}-t=0.35$. The plot scans the entire interfacial region between a bulk lamellar phase (left side where $g \rightarrow 2, f \rightarrow-1$ ) and the disordered phase (right side where $g \rightarrow 0, f \rightarrow 1$ ). (b) Analytical interfacial profile $\phi(x)$ using the same functions $f(x)$ and $g(x)$ of (a). The value of the average concentration in the two coexisting phases is: $\phi_{0}=0.25$ for the lamellar phase and $\phi_{0}=0.50$ for the disordered one.

Fig. 4 Numerical interfacial profile for $\varepsilon=0.35$ and $\mu=0.322$. In order to demonstrate that the most dominant bulk mode remains unchanged even in the interfacial region, the bulk lamellar phase is plotted as a dotted line. The horizontal line just below $\phi_{0}=0.5$ denotes the average concentration of the bulk disordered phase.

Fig. 5 Numerical interfacial profile for $\varepsilon=0.03$ and $\mu=0.249$. The horizontal line just above $\phi_{0}=0.3$ denotes the average concentration of the bulk disordered phase. The width of the interface is much larger than $2 \pi / q^{*}$, and over 100 lamellae are located in this interfacial region.

Fig. 6 (a) Interfacial tension as function of $\varepsilon=t_{t c p}-t$ plotted on a log-log plot. The straight line is the analytical interfacial tension $\gamma$. The dots represent the numerical results as is discussed in the text. The linear dependence of both the analytical and numerical results are in accord with the general scaling behavior expected close to the tricritical point. (b) Same results as in (a) but plotted differently. The square root of the interfacial tension $\gamma$ is plotted as a function of $\varepsilon=t_{t c p}-t$. The linear dependence is another confirmation of the tricritical point scaling $\gamma \sim \varepsilon^{2}$ 


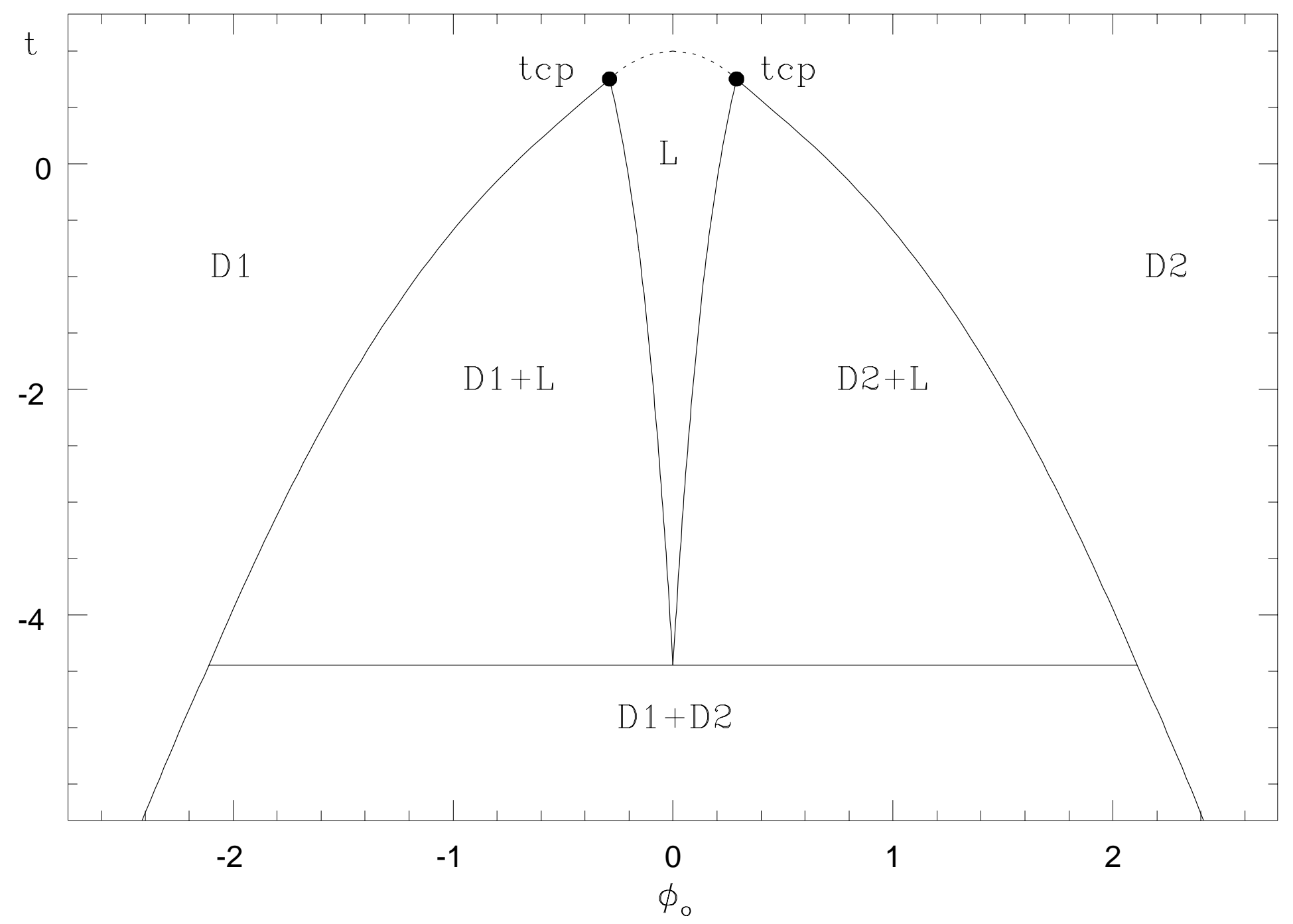




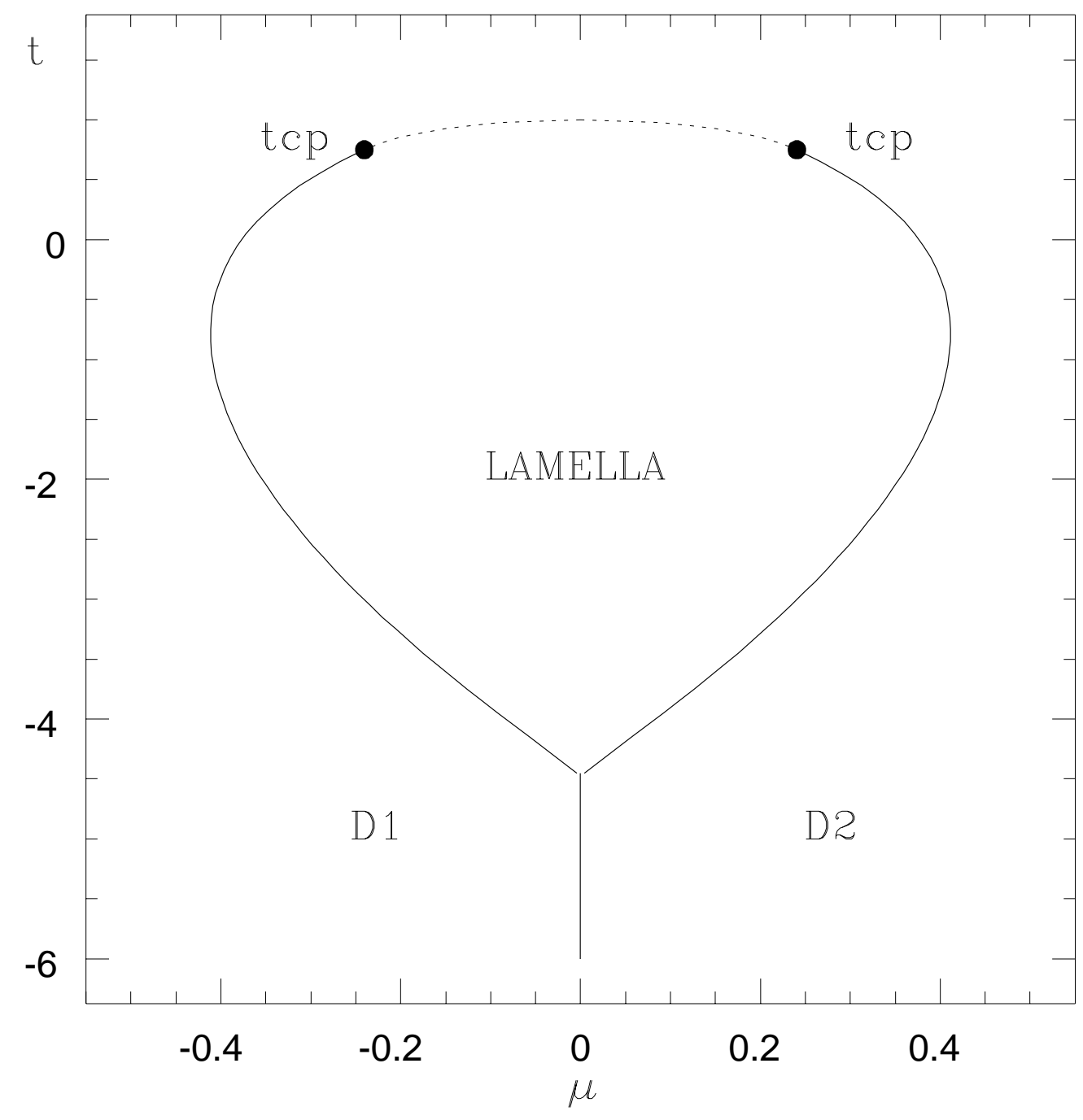




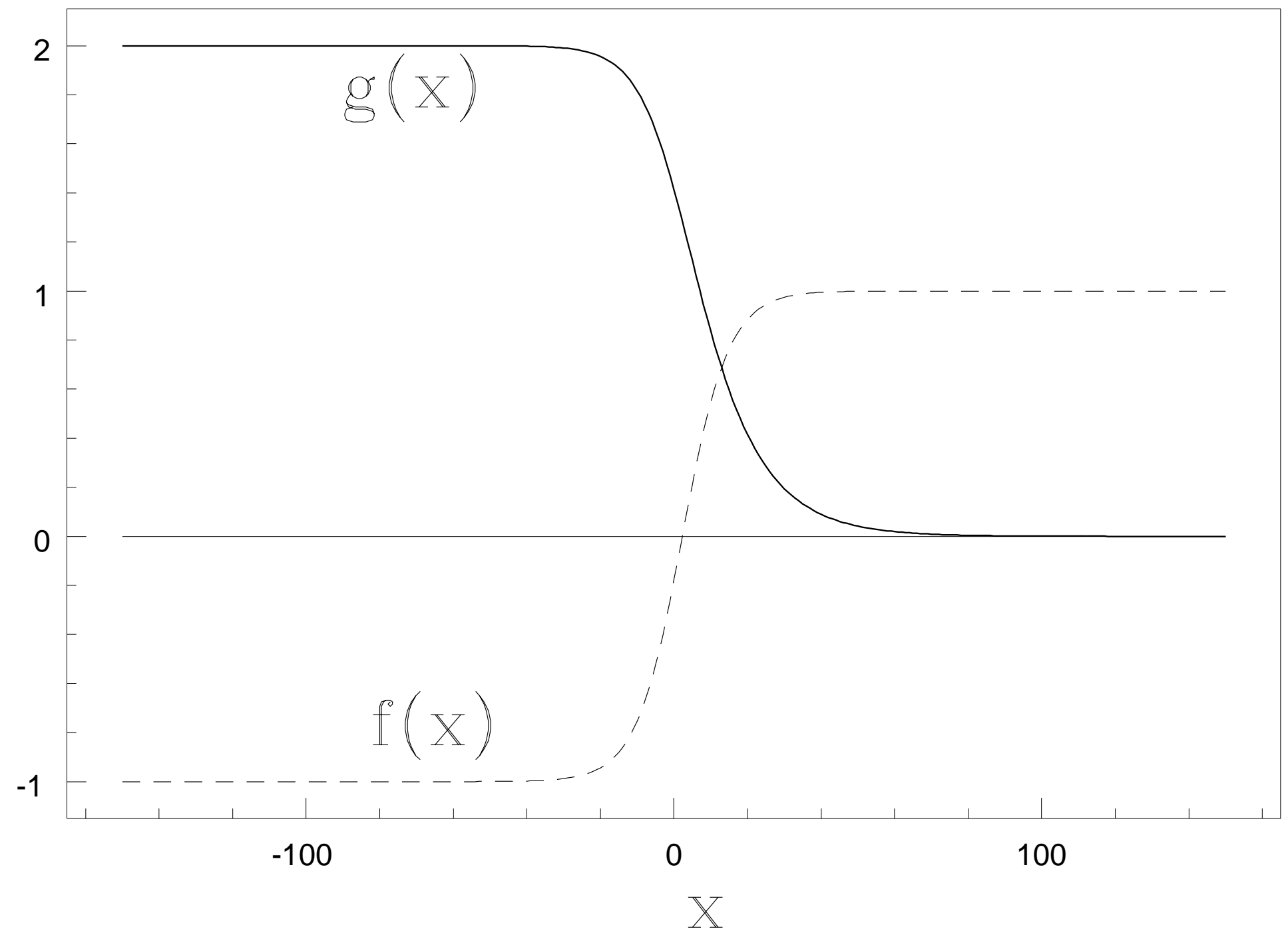




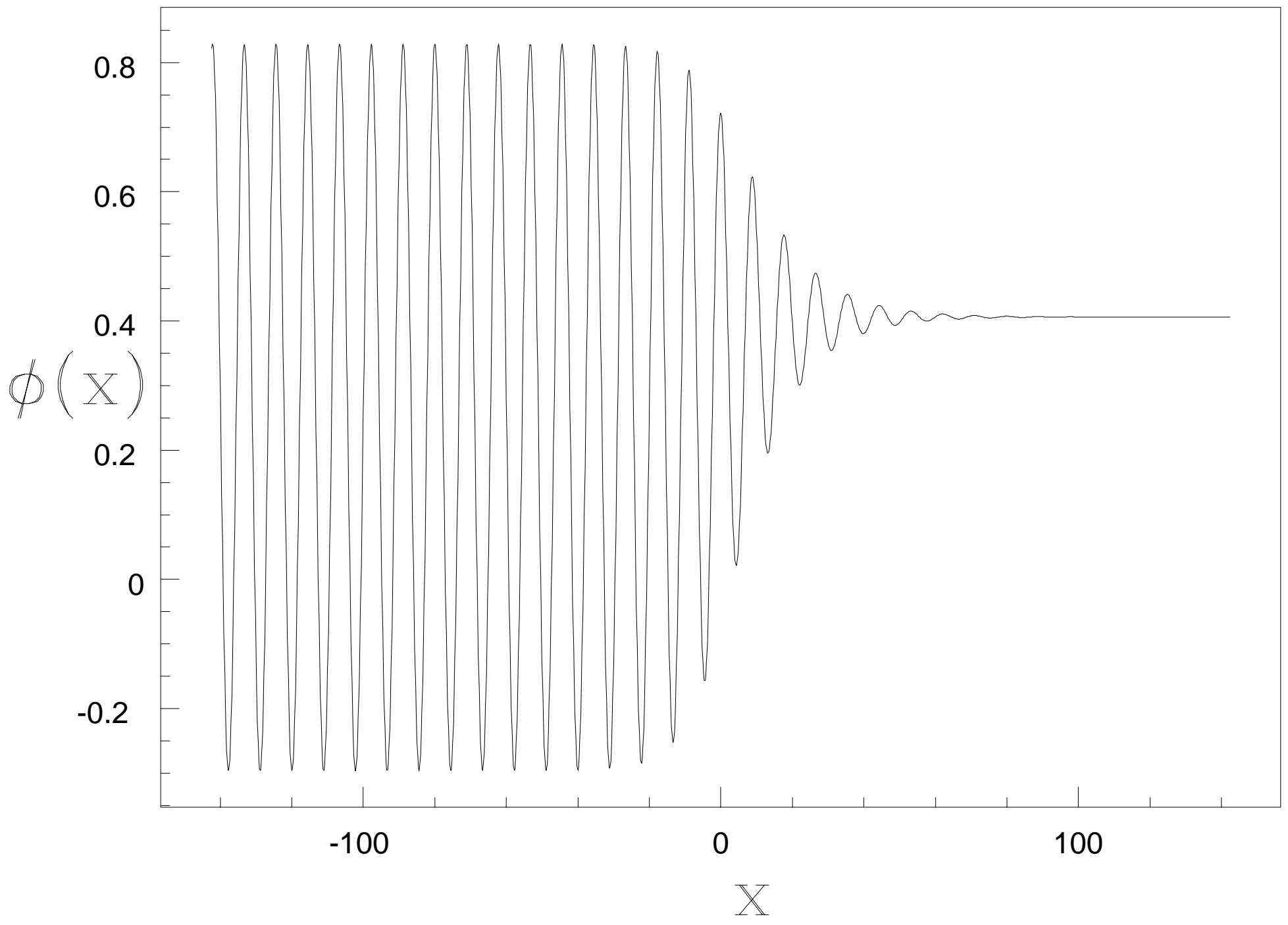




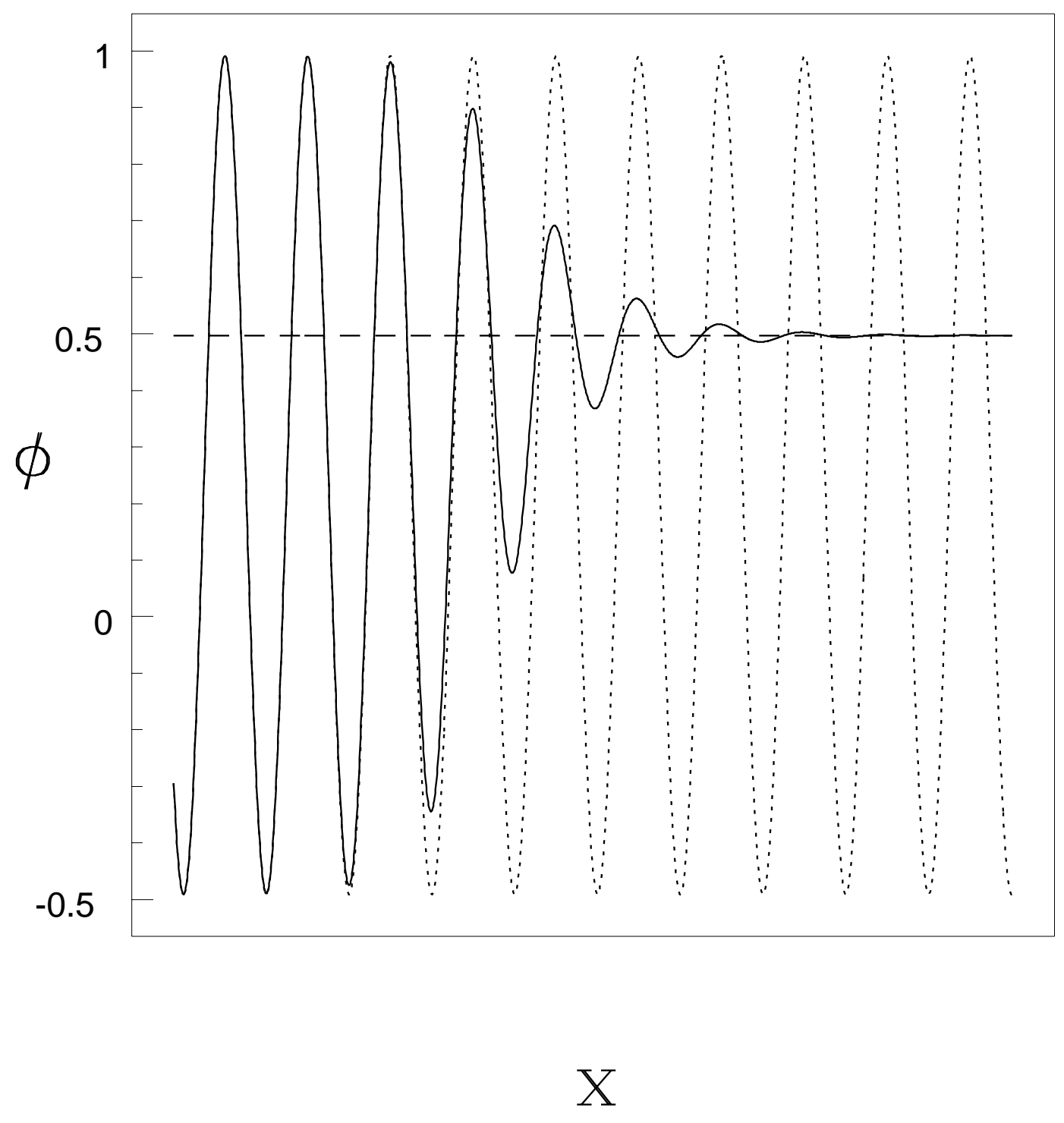




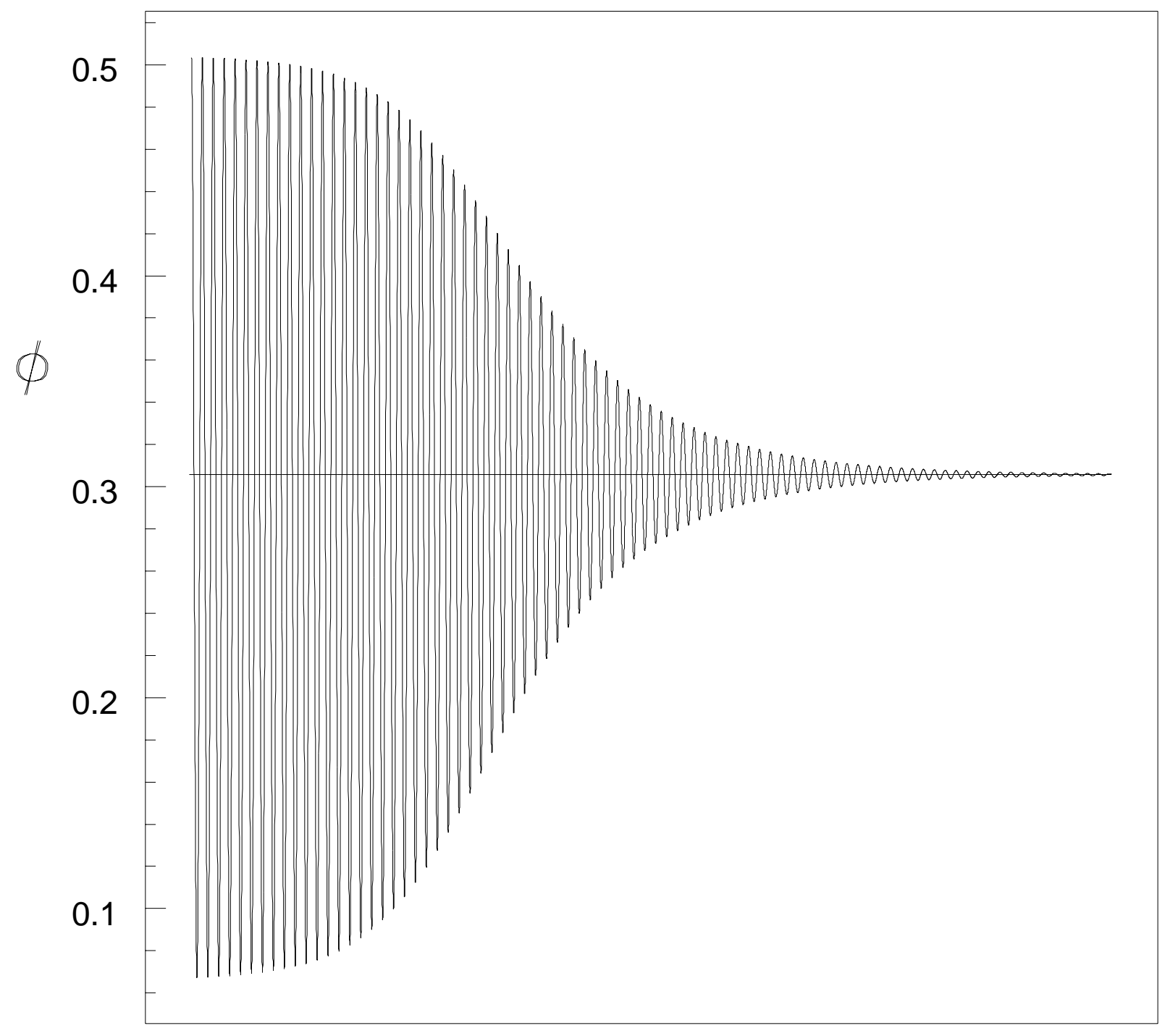

X 


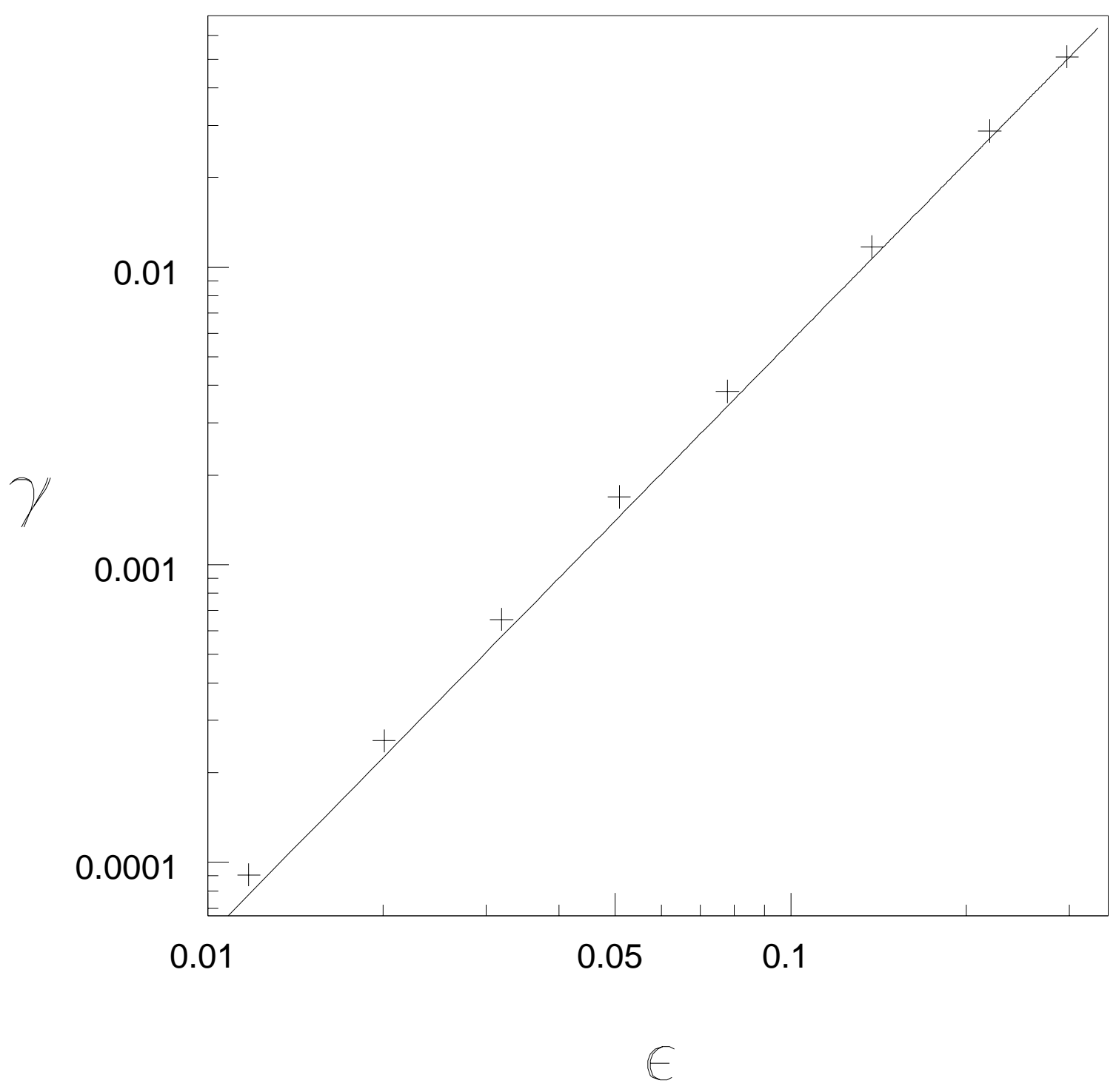




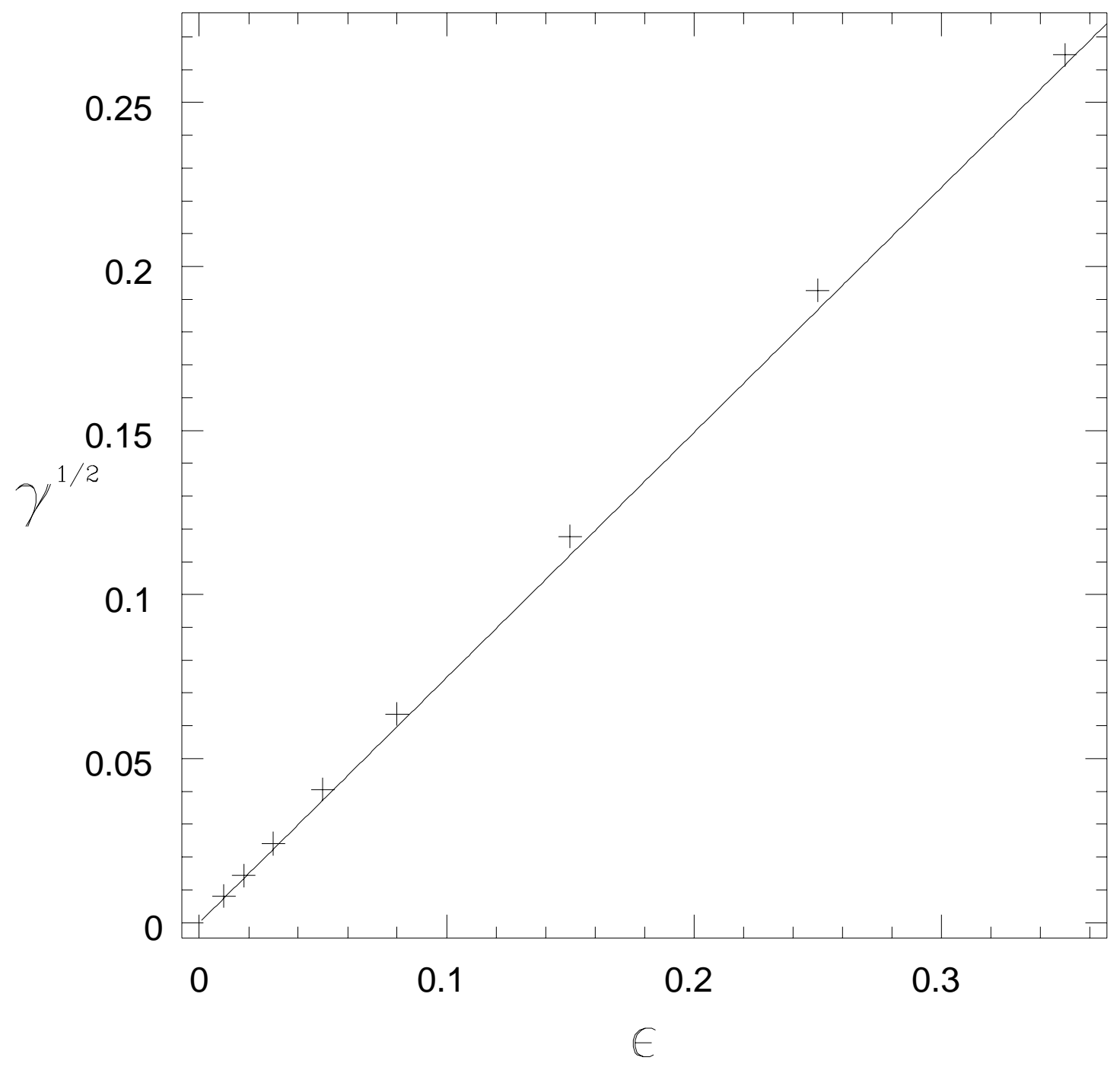

